\title{
Plasma cytokines interleukin-18 and C-X-C motif chemokine ligand 10 are indicative of the anti-programmed cell death protein-1 treatment response in lung cancer patients
}

\author{
Yida Wang ${ }^{1,2 \#}$, Hanxiao Chen ${ }^{3 \#}$, Tianzhuo Zhang ${ }^{1}$, Xue Yang $^{3}$, Jia Zhong ${ }^{3}$, Yuyan Wang ${ }^{3}$, Yujia Chi ${ }^{3}$, \\ Meina $\mathrm{Wu}^{3}$, Tongtong $\mathrm{An}^{3}$, Jianjie $\mathrm{Li}^{3}$, Xinghui Zhao ${ }^{3}, \mathrm{Zhi} \mathrm{Dong}^{3}$, Ziping Wang ${ }^{3}, \mathrm{Jun}_{\mathrm{Zhao}}{ }^{3}$, \\ Minglei Zhuo ${ }^{3}$, Jing Huang ${ }^{1,2} \wedge$
}

${ }^{1}$ Department of Immunology, School of Basic Medical Sciences, Peking University, and NHC Key Laboratory of Medical Immunology (Peking University), Beijing, China; ${ }^{2}$ Key Laboratory of Molecular Immunology, Chinese Academy of Medical Sciences, Beijing, China; ${ }^{3}$ Key Laboratory of Carcinogenesis and Translational Research (Ministry of Education/Beijing), Department of Thoracic Medical Oncology, Peking University Cancer Hospital \& Institute, Beijing, China

Contributions: (I) Conception and design: J Zhao, M Zhuo, J Huang; (II) Administrative support: J Zhao, M Zhuo, J Huang; (III) Provision of study materials or patients: All authors; (IV) Collection and assembly of data: All authors; (V) Data analysis and interpretation: Y Wang, H Chen, M Zhuo, J Huang; (VI) Manuscript writing: All authors; (VII) Final approval of manuscript: All authors.

\#These authors contributed equally to this work.

Correspondence to: Jun Zhao; Minglei Zhuo. Key Laboratory of Carcinogenesis and Translational Research (Ministry of Education/Beijing), Department of Thoracic Medical Oncology, Peking University Cancer Hospital \& Institute, 52 Fucheng Road, Haidian District, Beijing 100142, China. Email: ohjerry@163.com; minglei1978@163.com. Jing Huang. Department of Immunology, School of Basic Medical Sciences, Peking University Health Science Center, 38 Xueyuan Road, Haidian District, Beijing 100191, China. Email: huangjing82@bjmu.edu.cn.

\begin{abstract}
Background: Although programmed cell death protein-1 (PD-1)/programmed death ligand-1 (PD-L1) checkpoint inhibitors have shown prominent efficacy for treatment of advanced lung cancer, the outcomes of metastatic lung cancer remain poor throughout the world. Although progression-free survival (PFS) and overall survival (OS) have improved in the first- and second-line therapy settings for advanced lung cancer, the response rates to PD-1/PD-L1 inhibition range from 20\% to $40 \%$. Furthermore, patients may be at risk for immune-related adverse events (irAEs); hence, appropriate patient selection is crucial. This study aimed to identify a panel of plasma cytokines representing prognostic and predictive biomarkers of the response to anti-PD-1/PD-L1 treatment.

Methods: We prospectively studied 32 lung cancer patients who received anti-PD-1/PD-L1 antibody immunotherapy. Plasma cytokines in peripheral blood samples were evaluated and analyzed using flow cytometry at the time of diagnosis and at 2 months after the initiation of PD-1/PD-L1 inhibition.

Results: The baseline plasma concentrations of interleukin-18 (IL-18) and C-X-C motif chemokine ligand 10 (CXCL10) were correlated with the degree of tumor response. Moreover, the magnitude of plasma IL18 and CXCL10 level fluctuations were correlated significantly with the objective tumor response to antiPD-1/PD-L1 immunotherapy, and patients with high CXCL10 expression had significantly shorter PFS than those with low CXCL10 expression. A strong positive correlation between the fluctuation of IL-18 and interleukin-8 (IL-8) levels was detected, as was a negative correlation between the fluctuation of IL-18 and CXCL10 levels. The level of plasma C-C motif chemokine ligand 5 (CCL5) was significantly higher in patients with irAEs than in those without irAEs.

Conclusions: Plasma cytokines are related to the clinical efficacy of PD-1/PD-L1 inhibitors. IL-18 and CXCL10 are potential predictive markers for anti-PD-1/PD-L1 therapy in lung cancer patients and may play an important role in selecting patients who would benefit from PD-1/PD-L1 inhibitors.
\end{abstract}

\footnotetext{
^ ORCID: 0000-0002-0814-838X.
} 
Keywords: Cytokine; lung cancer; programmed cell death protein-1 (PD-1)/programmed cell death ligand-1 (PD-L1); interleukin-18; CXCL10

Submitted Feb 11, 2020. Accepted for publication Sep 30, 2020.

doi: $10.21037 /$ atm-20-1513

View this article at: http://dx.doi.org/10.21037/atm-20-1513

\section{Introduction}

Lung cancer is one of the most common malignancies worldwide, with considerable morbidity and mortality (1). In many countries, including China, lung cancer is the leading cause of cancer-related mortality. More than half of patients with non-small cell lung cancer (NSCLC) initially present with advanced stages of the disease (2).

Immune checkpoints are the $T$ cell regulatory mechanisms of co-stimulatory and inhibitory signals that control the amplitude and quality of the immune response (3). The activation of programmed cell death protein-1 (PD-1)/programmed cell death ligand-1 (PDL1) has been recognized as a major immunoinhibitory mechanism in solid tumors (4). Accordingly, the anti-PD-1 antibodies nivolumab and pembrolizumab, and the antiPD-L1 antibody atezolizumab, have shown remarkable clinical benefits in various solid tumors, resulting in the approval of these agents by the FDA for patients with NSCLC (5-7). Moreover, favorable responses using PD-1/ PD-L1 inhibitors as monotherapy or in combination with chemotherapy have been reported (7). Nonetheless, these immune checkpoint inhibitors elicit durable clinical responses in only a fraction of patients. Moreover, the objective response rates (ORRs) of nivolumab or pembrolizumab were reported to be approximately $20 \%$ in patients with previously treated NSCLC $(5,6)$.

Although PD-L1 expression is a logical biomarker for predicting response to anti-PD-1/PD-L1 immunotherapies (8), the predictive value of PD-L1 expression for immunotherapy is currently under debate. Moreover, because of the difficulty associated with obtaining tumor tissues, the identification of prognostic biomarkers in circulating blood for patient selection in pragmatic clinical settings would be of great value for optimizing and personalizing anti-PD-1/PD-L1 immunotherapy. We previously reported that circulating $\mathrm{T}$ cell subtypes may reflect the outcome of immunotherapy in lung cancer patients (9).

Moreover, an increasing number of preclinical and clinical studies have suggested that infiltrating immune cells within a tumor or the tumor cells themselves produce cytokines and chemokines, leading to a modulation of the tumor microenvironment and promoting angiogenesis, growth, invasion and metastasis (10). In this study, we aimed to investigate the association between plasma cytokine levels and the response to anti-PD-1/PD-L1 treatment, and to further assess the value of cytokine levels as predictive biomarkers for lung cancer patients treated with PD-1/PD-L1 inhibitors.

We present the following article in accordance with the STROBE reporting checklist (available at http://dx.doi. org/10.21037/atm-20-1513).

\section{Methods}

\section{Patients}

A total of 32 patients with a histologically confirmed diagnosis of stage III/IV advanced lung cancer were enrolled in the immunotherapy cohort of this study between September 2017 and August 2018. Patients with a history of interstitial lung disease, systemic immunosuppressive therapy, or active autoimmune disease were excluded. Twenty-three patients who were diagnosed with stage III/ IV NSCLC and treated with platinum and gemcitabine/ pemetrexed chemotherapy (chemotherapy cohort) and 20 healthy controls were also enrolled in this study, and their clinical features are summarized in Table 1.

This study was conducted in accordance with the Declaration of Helsinki (as revised in 2013). The study was approved by the Institutional Ethics Board of Peking Cancer Hospital (No. 2017KT57) and informed consent was obtained from all the patients.

\section{Response evaluation}

Clinical examinations, CT scans of the chest and abdomen, cerebral MRI/CT and lymph node ultrasound scans were performed at baseline and then every 6 weeks. For all patients, tumor burden and the overall response were assessed according to the modified Response Evaluation 
Table 1 Clinicopathologic characteristics of the study population

\begin{tabular}{|c|c|c|c|c|}
\hline & \multicolumn{3}{|c|}{ Immunotherapy ( $\mathrm{n=32}$ ) } & $\frac{\text { Chemotherapy }(n=23)}{\text { Platinum \& Gemcitabine/Pemetrexed }}$ \\
\hline \multicolumn{5}{|l|}{ Age (years) } \\
\hline Mean \pm SD & $60 \pm 5.7$ & $52.8 \pm 1.3$ & $58.3 \pm 7.8$ & $60.2 \pm 10.3$ \\
\hline \multicolumn{5}{|l|}{ Sex } \\
\hline Female & $4(23.5)$ & $2(20 \%)$ & $2(40 \%)$ & $7(30 \%)$ \\
\hline \multicolumn{5}{|l|}{ Smoking status } \\
\hline Current or former smoker & $9(52.9 \%)$ & $6(60 \%)$ & $4(80 \%)$ & $13(57 \%)$ \\
\hline Never smoker & $8(47.1 \%)$ & $4(40 \%)$ & $1(20 \%)$ & $10(43 \%)$ \\
\hline Squamous carcinoma & $3(17.6 \%)$ & $4(60 \%)$ & $2(40 \%)$ & $4(16 \%)$ \\
\hline Adenosquamous carcinoma & $1(5.9 \%)$ & $0(0 \%)$ & $0(0 \%)$ & $1(4 \%)$ \\
\hline Small cell lung carcinoma & $2(11.8 \%)$ & $0(0 \%)$ & $1(20 \%)$ & $0(0 \%)$ \\
\hline \multicolumn{5}{|l|}{ Disease stage } \\
\hline III & $5(29.4 \%)$ & $3(30 \%)$ & $2(40 \%)$ & $7(30 \%)$ \\
\hline IV & $12(70.6 \%)$ & $7(70 \%)$ & $3(60 \%)$ & $16(70 \%)$ \\
\hline \multicolumn{5}{|l|}{ PD-L1 expression } \\
\hline$\geq 1 \%$ & $7(41.2 \%)$ & $1(10 \%)$ & $0(20 \%)$ & ND \\
\hline Progressive disease & $8(47.1 \%)$ & $4(40 \%)$ & $0(0 \%)$ & $6(26 \%)$ \\
\hline \multicolumn{5}{|l|}{ irAE } \\
\hline Yes & $11(64.7 \%)$ & $3(30 \%)$ & $3(60 \%)$ & ND \\
\hline No & $6(35.3 \%)$ & $7(70 \%)$ & $2(40 \%)$ & ND \\
\hline
\end{tabular}

ND, not detected; irAE, immune related adverse events.

Criteria in Solid Tumors (RECIST 1.1) and categorized as complete response (CR), partial response (PR), stable disease (SD), and progressive disease (PD) (11). The patients who were diagnosed with disease progression at the initial immunotherapy session underwent a confirmation evaluation, including CT/MRI/Ultrasonic examinations,
8 weeks later to exclude pseudoprogression. Immunerelated adverse events (irAEs) were continuously monitored and evaluated in all treated patients and graded according to the National Cancer Institute Common Terminology Criteria for Adverse Events, version 3.0. irAEs were managed following protocol-specific guidelines. 


\section{Sample collection}

Enrolled patients received either pembrolizumab or atezolizumab every 3 weeks, or nivolumab every 2 weeks intravenously until disease progression. No treatment was stopped because of unacceptable toxicity. Relevant clinical information and blood samples were collected before and after two cycles of anti-PD-1/PD-L1 treatment or chemotherapy. Peripheral blood samples were obtained by venipuncture ( $10 \mathrm{~mL}$; BD Vacutainer glass serum tube) and centrifuged (2,000 g, 20 minutes) to isolate the plasma, which was stored at $-80{ }^{\circ} \mathrm{C}$ in aliquots until analysis. The time interval between processing and freezing was no more than $2 \mathrm{~h}$ for each sample. No freeze-thaw cycles were performed before analysis.

\section{Cytometric bead array immunoassay analysis of cytokines}

The levels of various cytokines in plasma samples were measured with LEGENDplex ${ }^{\mathrm{TM}}$ Human Inflammation and Proinflammatory Chemokine Panel Kits from BioLegend (Cat. \#740102 and \#740003), which allow the simultaneous quantification of 13 human cytokines in a single sample to provide higher detection sensitivity and a broader dynamic range than the traditional ELISA method. All steps in the assay were conducted according to the manufacturer's instructions. Specifically, $25 \mu \mathrm{L}$ of plasma, $25 \mu \mathrm{L}$ of beads and $25 \mu \mathrm{L}$ of detection antibodies from the panel kit were mixed and incubated for $2 \mathrm{~h}$ at room temperature. Samples were incubated with streptavidin-phycoerythrin for another $30 \mathrm{~min} ; 200 \mu \mathrm{L}$ of wash buffer was added, and the samples were centrifuged for $5 \mathrm{~min}$. A BD FACSVerse (BD Biosciences) system was calibrated with setup beads, and 3,000 events were acquired for each sample. Various cytokine concentration ratios were measured according to the fluorescence intensities of individual cytokines, and cytokine levels below the detection limit were assigned a value equal to the lowest detectable value of that cytokine. The cytokines examined were as follows: interleukin-1 beta (IL-1ß), IL-6, IL-10, IL-12p70, IL-17A, IL-18, IL-23, IL-33, interferon alpha 2 (IFN- $\alpha 2)$, interferon gamma (IFN- $\gamma$ ), tumor necrosis factor alpha (TNF- $\alpha$ ), C-C motif chemokine ligand 2 (CCL2, also named MCP-1), CCL3 (MIP-1 $\alpha$ ), CCL4 (MIP-1ß), CCL5 (RANTES), CCL11 (EOTAXIN), CCL17 (TARC), CCL20 (MIP-3 $\alpha$ ), C-X-C motif chemokine ligand 1 (CXCL1, also named GRO- $\alpha$ ), CXCL5 (ENA-78), CXCL8 (IL-8), CXCL9 (MIG), CXCL10 (IP-10), and CXCL11 (I-TAC).

\section{Statistical analysis}

All measurements were performed in duplicate. All values are expressed as the mean \pm standard error of the mean (SEM). All statistical analyses were performed using Prism 6.0 statistical software (GraphPad Software Inc.). Two-way analysis of variance (ANOVA) was employed to compare differences between groups. Two-tailed Student's $t$-tests were used when only two groups were analyzed. Pearson's correlation coefficient (Rsq) was applied to assess the reproducibility of the assay between near-serial sections of the index array. Linear regression coefficients were calculated to determine associations between the levels of different cytokines. Progression-free survival (PFS) was estimated using the Kaplan-Meier method and compared with a nonparametric log-rank test. A multivariate Cox proportional hazard regression model was applied with potential risk factors as covariates, and hazard ratios (HRs) and their $95 \%$ confidence intervals (CIs) were obtained. A $\mathrm{P}$ value was considered statistically significant at $<0.05$.

\section{Results}

\section{Patient characteristics}

The immunotherapy cohort included 19 cases of adenocarcinoma, 9 cases of squamous carcinoma, 1 case of adenosquamous carcinoma and 3 cases of small cell lung cancer. The median age of the patients was 58 years (range, 41-69 years), with a male:female ratio of 3:1. The clinical staging was as follows: stage III $(31.3 \%)$ and stage IV (68.7\%). In total, 19 (59.4\%) patients were current or former smokers (Table 1). These patients received antiPD-1/PD-L1 treatments for a median of 8 cycles, which corresponded to a median treatment period of 6 months, and 12 patients were still under treatment when this article was written. Atezolizumab was administered to $17 / 32$ (53.13\%) patients, nivolumab was administered to $10 / 32$ (31.25\%) patients, and pembrolizumab was administered to the remaining 5/32 (15.62\%) patients. Among the 10 patients for whom PD-L1 expression was detected, 8 were positive for PD-L1 expression (mostly patients treated with atezolizumab). Seventeen (53.1\%) patients experienced irAEs, with rash and fatigue as the most prevalent. Overall, grade 1 irAEs were reported in 8 patients, grade 2 irAEs in 5 patients, and grade 3 irAEs in 4 patients. No grade 4 to 5 irAEs were reported.

At the time of analysis, the median PFS for all patients was 3.8 months (95\% CI, 2.81-6.12), and the median 

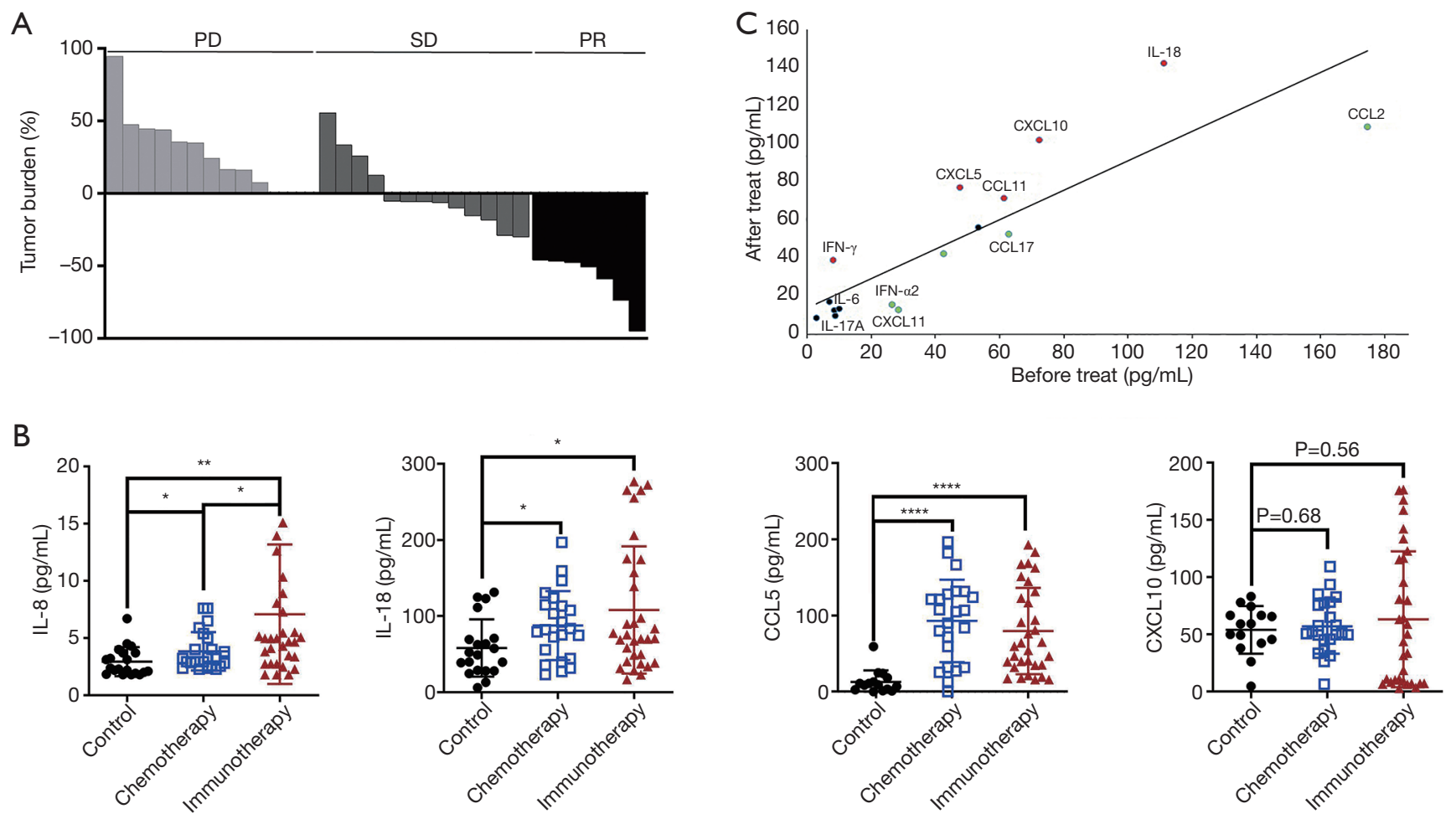

Figure 1 Levels of plasma cytokines on anti-PD-1/PD-L1 monotherapy were shown. (A) Waterfall plot for the best tumor response in lung cancer patients on anti-PD-1/PD-L1 monotherapy $(\mathrm{n}=32)$. (B) Expression levels of plasma IL-8, IL-18, CCL5 and CXCL10 in lung cancer with immunotherapy $(n=32)$, chemotherapy $(n=23)$ and healthy controls $(n=20)$. (C) Comparison of the average concentration of 16 cytokines in the plasma of lung cancer patients prior to and during antiPD-1/PD-L1 treatment $(\mathrm{n}=32)$. *, $\mathrm{P}<0.05$; **, $\mathrm{P}<0.01$; ****, $\mathrm{P}<0.0001$. Red, increased cytokines; black, not changed cytokines; green, reduced cytokines. $\mathrm{PD}$, progressive disease; $\mathrm{SD}$, stable disease; $\mathrm{PR}$, partial response.

overall survival (OS) was not reached. Patients were divided into PR (7/32, 21.9\%), SD (13/32, 40.6\%) and PD (12/32, $37.5 \%)$ subgroups according to the best therapy response (Figure 1A).

The chemotherapy cohort included 23 NSCLC patients: 18 cases of adenocarcinoma, 4 cases of squamous carcinoma and 1 case of adenosquamous carcinoma. The median age of the patients was 60.2 years, with a male:female ratio of $2.3: 1$. The clinical staging was as follows: stage III $(30 \%)$ and stage IV (70\%). In total, 13 (57\%) patients were current or former smokers (Table 1). These patients received platinum and gemcitabine/pemetrexed chemotherapy. Moreover, 20 healthy controls (those without chronic diseases), with a median age of 50.2 years and a male:female ratio of $3: 2$, were enrolled in the cohort study.

\section{Levels of multiple cytokines in plasma at baseline and during treatment}

To understand the immune response in patients and identify immune correlations with the objective tumor response, 24 immune-related cytokines and chemokines were measured in plasma samples. Among them, certain ILs, including IL-6, IL-8 and IL-18, are reported to be promising for cancer immunotherapy. Furthermore, IFNs and chemokines, such as IFN- $\gamma$ and CXCL10, are potent immunomodulators that shape immunity through direct actions on innate and adaptive immune cells. Because of the limited sensitivity of the BioPlex system under our experimental conditions, 17 types of cytokines were detected in this study (Table 2). 


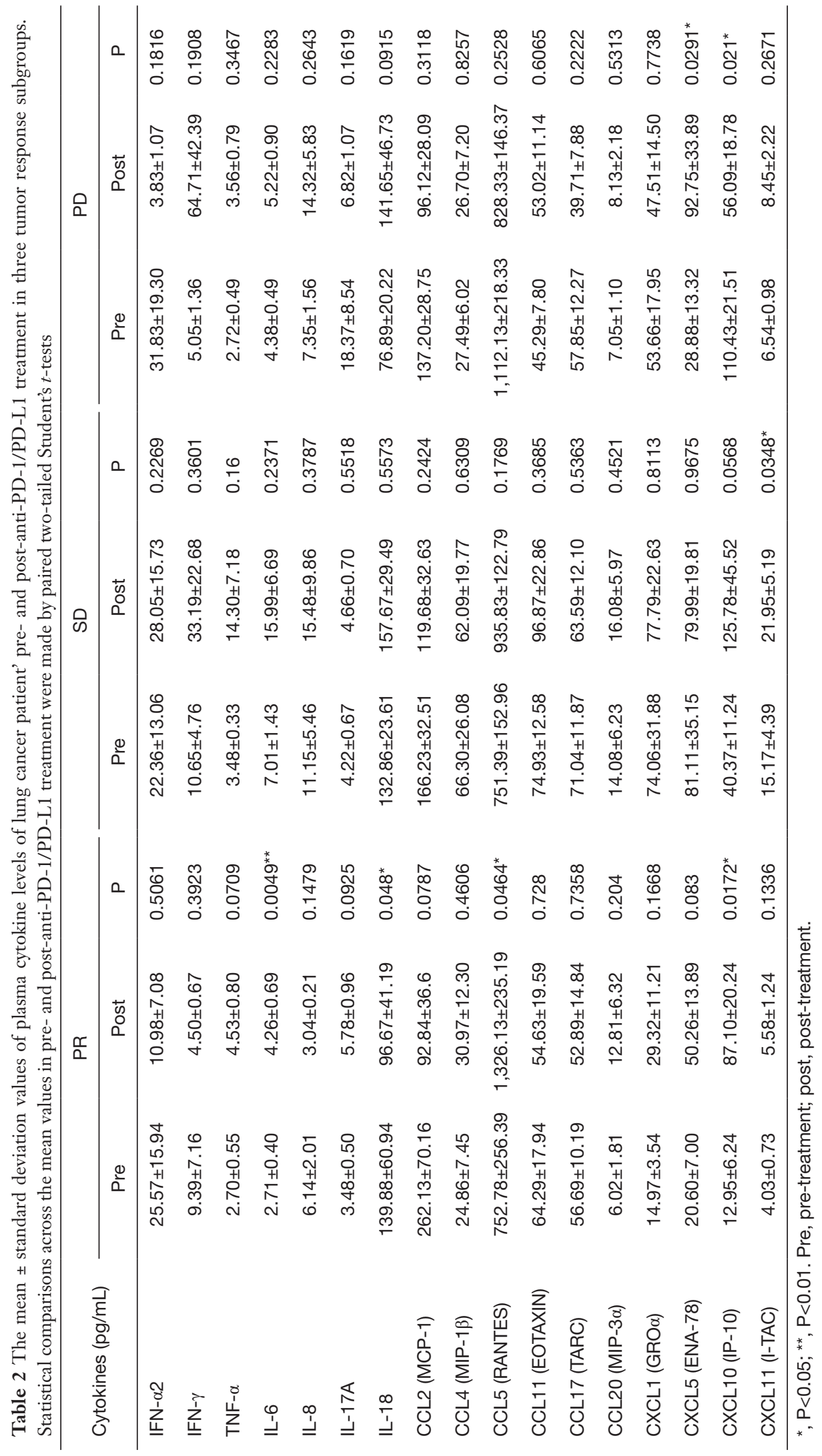



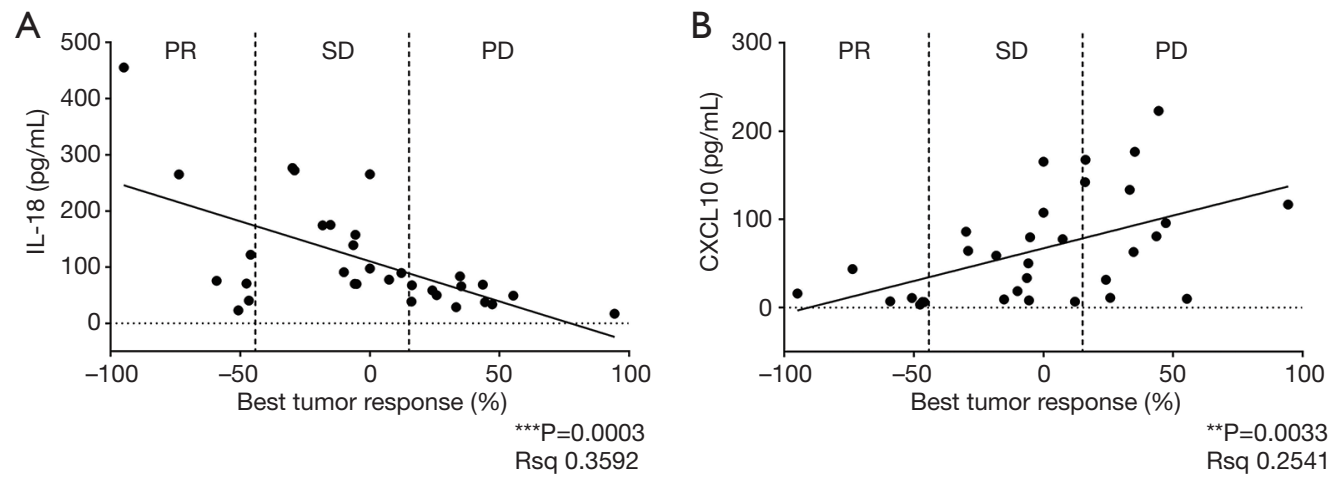

Figure 2 Correlation between the best tumor response and baseline plasma IL-18 (A) or CXCL10 (B) levels using twotailed Pearson's correlation analysis. Patients divided into partial response $(\mathrm{PR}, \mathrm{n}=7)$, stable disease $(\mathrm{SD}, \mathrm{n}=13)$ or progressive disease (PR, $\mathrm{n}=12)$ subgroups.

We firstly compared the baseline plasma cytokine levels between lung cancer patients and healthy controls. As shown in Figure 1B, at baseline, the plasma levels of IL18 , IL-8, and especially CCL5 were higher in lung cancer patients than in healthy controls, while no significant difference in the plasma CXCL10 level at baseline was found between lung cancer patients and healthy controls.

Second, a comparison of the average concentration of 17 cytokines in the plasma of lung cancer patients prior to and during treatment was conducted. In the immunotherapy cohort, the cytokines CCL2, CCL5 and IL-18 were generally highly detected. Th1 cytokines (IFN- $\gamma$, IL-18, and CXCL10) were significantly increased in response to anti-PD-1/PD-L1 treatment, and CXCL5 and CCL11 were moderately elevated; in contrast, IFN- $\alpha 2$, CCL2 and CCL17 were decreased. Moreover, anti-PD-1/PDL1 treatment led to a reduction in the Th17-related level of cytokine IL-17A, although the IL-6 level was not consistently altered (Figure 1C). In our multiplex analysis of cytokine levels pre- and posttreatment, significant changes in five cytokines were observed (IL-6, IL-18, CCL5, CXCL5 and CXCL10) in different response subgroups $(\mathrm{P}<0.05)$ (Table 2).

\section{Analysis of the correlation between plasma cytokines and the tumor response}

We first assessed the relationship between the levels of cytokines and the objective tumor response in the immunotherapy cohort. At baseline, the level of IL18 showed a positive correlation with tumor remission $(\mathrm{P}=0.0003$, Figure $2 A)$, whereas the level of CXCL10 showed a negative correlation with tumor regression $(\mathrm{P}=0.0033$, Figure 2B). However, there was no correlation between other cytokine levels at baseline and the tumor response.

Next, we evaluated the correlation of the tumor response and fluctuations in cytokine levels (changes in cytokine levels from pre- to posttreatment) (Table 3) and found that changes in CCL5 and CXCL10 were strongly associated with patient response. In addition, the levels of IL-6, IL18, CXCL10 and CCL5 were correlated with PR, and the levels of CXCL5 and CXCL10 were correlated with PD. The IL-18 level decreased in patients who benefited from anti-PD-1/PD-L1 treatment but clearly increased in those with disease progression. In contrast, the levels of CCL5 and CXCL10 increased in the PR and SD subgroups but decreased in the PD subgroup (Table 2). The levels of IL-18, CXCL10 and CCL5 before and after treatment categorized by different responses are shown in Figure 3. We also analyzed the changes in plasma cytokines in patients treated with pembrolizumab, nivolumab or atezolizumab and found that the changes in IL-18, CCL5 and CXCL10 were similar among the different treatment subgroups even though the number of patients in the pembrolizumab subgroup was too small for statistical analysis (data not shown).

However, in the chemotherapy cohort, neither the baseline levels nor dynamic changes of IL-18, CXCL10, IL-8 or CCL5 were significantly correlated with the tumor response. In addition, the changes in IL-18, CXCL10 and CCL5 levels were similar between the adenocarcinoma and squamous carcinoma subgroups. Moreover, we performed a multivariate analysis of the changes in plasma cytokines and histology and found no significant difference between the different subtypes, although the changes in the 
Table 3 Fluctuations in different cytokine levels from pre- to post- anti-PD-1/PD-L1 treatment, their mean values, standard deviation and their statistical correlation with patient response

\begin{tabular}{|c|c|c|c|}
\hline & Mean value $(\mathrm{pg} / \mathrm{mL})$ & Standard deviation $(\mathrm{pg} / \mathrm{mL})$ & $\mathrm{P}$ value (correlation with response) \\
\hline IFN- $\gamma$ & 30.46 & 112.78 & 0.4308 \\
\hline TNF- $\alpha$ & 5.11 & 23.58 & 0.2663 \\
\hline IL-6 & 4.64 & 159.53 & 0.5020 \\
\hline IL-17A & -3.72 & 22.36 & 0.1327 \\
\hline IL-18 & 23.59 & 17.94 & 0.1788 \\
\hline CCL2 & -68.19 & 122.95 & 0.2084 \\
\hline CCL4 & -0.67 & 127.03 & 0.6401 \\
\hline CCL17 & -10.66 & 689.95 & 0.7320 \\
\hline CCL20 & 2.76 & 95.74 & 0.4154 \\
\hline CXCL1 & 2.34 & 16.76 & 0.7557 \\
\hline CXCL5 & 29.99 & 57.39 & 0.1836 \\
\hline CXCL10 & 34.35 & 16.75 & $0.0098^{\star *}$ \\
\hline CXCL11 & 3.77 & 24.81 & 0.1875 \\
\hline
\end{tabular}

Cytokine level changes from pre- to post-treatment measurements. *, $\mathrm{P}<0.05 ;{ }^{\star \star}, \mathrm{P}<0.01$.

A

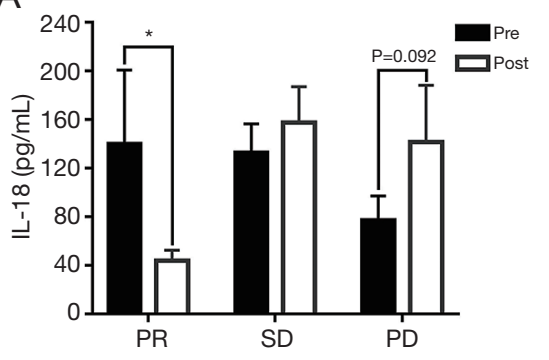

B

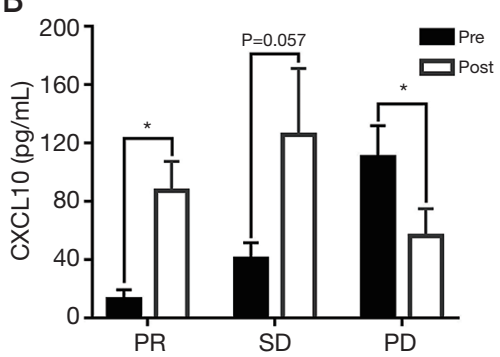

C

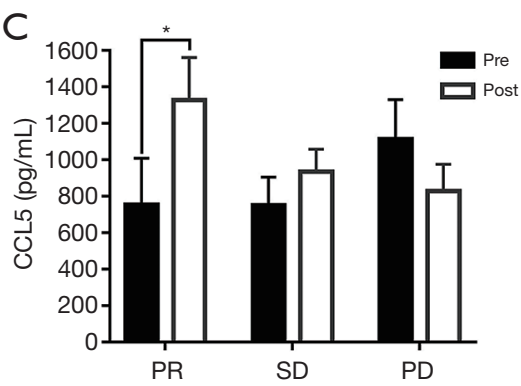

Figure 3 Induction of plasma IL-18 (A), CXCL10 (B) and CCL5 (C) reflects objective tumor response to PD-1/PD-L1 inhibitors. Significant differences in cytokine levels were detected between plasma samples pretreatment and posttreatment according to two-tailed student's $t$-tests. Data are represented as means \pm SEM. Pre, pretreatment; Post, posttreatment. Partial response ( $\mathrm{PR}, \mathrm{n}=7$ ), stable disease $(\mathrm{SD}, \mathrm{n}=13)$ and progressive disease (PD, $\mathrm{n}=12)$. *, $\mathrm{P}<0.05$.

adenocarcinoma subgroup were more obvious than those in the squamous carcinoma subgroup (data not shown). All these data suggest that the degree of plasma IL-18 and CXCL10 induction is significantly associated with the tumor response to anti-PD-1/PD-L1 therapy.

\section{Analysis of the association between PFS and plasma CXCL10 and IL-18}

PFS was assessed in all patients treated with anti-PD-1/ PD-L1 antibody therapies using receiver operating characteristic (ROC) curves. The area under the curve 

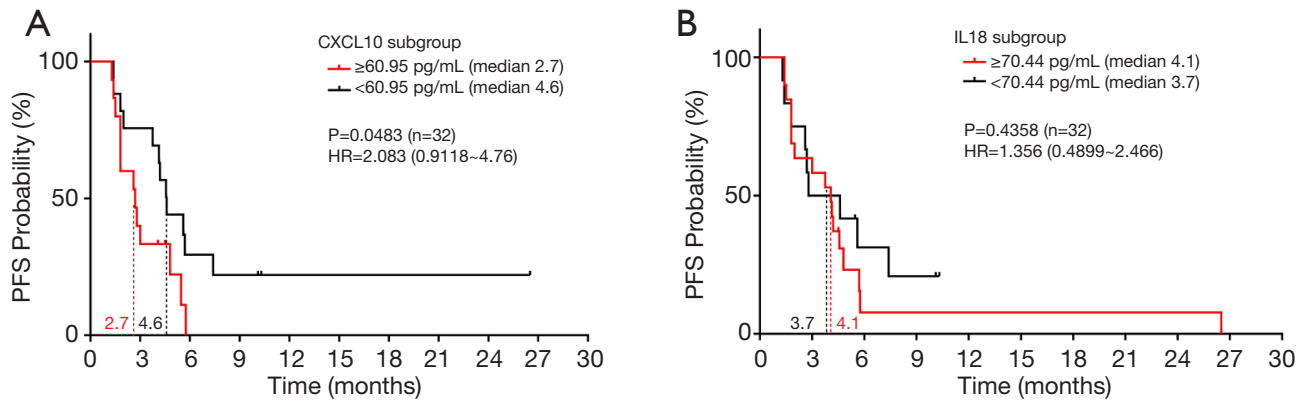

Figure 4 Association between elevated CXCL10 level and reduced PFS was observed in lung cancer patients treated with PD-1/PDL1 inhibitors ( $\mathrm{n}=32$ ). The 2-sided log-rank test $\mathrm{P}$ values for the survival analysis, the PFS HR (based on Cox proportional hazard model) between CXCL-10 and IL-18 subgroups are indicated. (A) Probability of PFS with $\geq 60.95$ versus $<60.95 \mathrm{pg} / \mathrm{mL}$ CXCL10 levels. (B) Probability of PFS with $\geq 70.44$ versus $<70.44 \mathrm{pg} / \mathrm{mL}$ IL-18 levels. HR, hazard ratio.

(AUC) identified plasma CXCL10 levels $\geq 60.95 \mathrm{pg} / \mathrm{mL}$ and plasma IL-18 levels $\geq 70.44 \mathrm{pg} / \mathrm{mL}$ as cut-off values for PFS. Based on the optimal cut-off values as stratification points, we further compared the association between PFS and CXCL10 subgroups ( $\geq 60.95$ versus $<60.95 \mathrm{pg} / \mathrm{mL}$ ) or IL-18 subgroups ( $\geq 70.44$ versus $<70.44 \mathrm{pg} / \mathrm{mL}$ ) using two-sided log-rank tests. High baseline CXCL10 level was significantly associated with poor PFS (HR $=2.083,95 \%$ CI: 0.9118-4.76 months, $\mathrm{P}=0.0483$ ) (Figure $4 A$ ), suggesting that CXCL10 could be a biomarker to predict a subset of patients who are likely to benefit from anti-PD-1/PD-L1 therapy.

However, no significant association between an elevated baseline plasma IL-18 level and prolonged PFS was observed (HR $=1.356,95 \%$ CI: 0.4899-2.466 months, $\mathrm{P}=0.4358$ ) (Figure $4 B$ ), suggesting that IL18 might be a predictive marker indicating partial remission but not survival for these patients. In the chemotherapy cohort, there was no significant association between baseline CXCL10 or IL-18 levels and the PFS of NSCLC patients receiving chemotherapy treatment (Figure S1), suggesting the predictive value of plasma CXCL10 for anti-PD-1/PDL1 immunotherapy.

\section{Assessment of the objective response to anti-PD-1/PD- L1 treatment by analyzing associations between different plasma cytokines}

The associations between fluctuations in the levels of different plasma cytokines were evaluated using linear regression analysis, and a strong positive correlation of IL18 and IL-8 $(\mathrm{P}=0.0009)$ and a negative correlation of IL-18 and CXCL10 $(\mathrm{P}=0.001)$ were identified (Figure 5). Although
IL-18 and CXCL10 induction was significantly associated with the tumor response, a significant relationship was not observed when IL-18 and CXCL10 or IL-8 were combined.

\section{Associations between irAEs and plasma cytokine levels}

We explored the value of plasma cytokines in predicting the risk of irAEs. At baseline, there was no notable difference in plasma cytokines between patients with irAEs and those without irAEs, while CCL5 but not IL-18 and CXCL10 differed between these two subsets of patients after 2 cycles of anti-PD-1/PD-L1 treatment (Figure 6). Moreover, the level of CCL5 was significantly associated with irAE onset, suggesting that CCL5 might be involved in irAE onset caused by anti-PD-1/PD-L1 therapy.

\section{Discussion}

To date, reliable predictive markers for PD-1/PD-L1 inhibitors in lung cancer are still under investigation (12). In this study, we prospectively evaluated lung cancer patients who received anti-PD-1/PD-L1 therapy. First, we observed significant changes in the plasma levels of IL-18, CCL5 and CXCL10 after immunotherapy $(\mathrm{P}<0.05)$. More importantly, we found that both the baseline concentration and fluctuations of plasma IL-18 and CXCL10 levels were correlated significantly with the objective tumor response to anti-PD-1/PD-L1 immunotherapy. We also found that patients with high CXCL10 expression had significantly shorter PFS than those with low CXCL10 expression.

Previous studies have investigated plasma cytokines at baseline, including IFN- $\gamma(1.23 \pm 0.430 \mathrm{pg} / \mathrm{mL})$ and TNF- $\alpha(1.215 \pm 0.3663 \mathrm{pg} / \mathrm{mL})$, in patients with advanced 

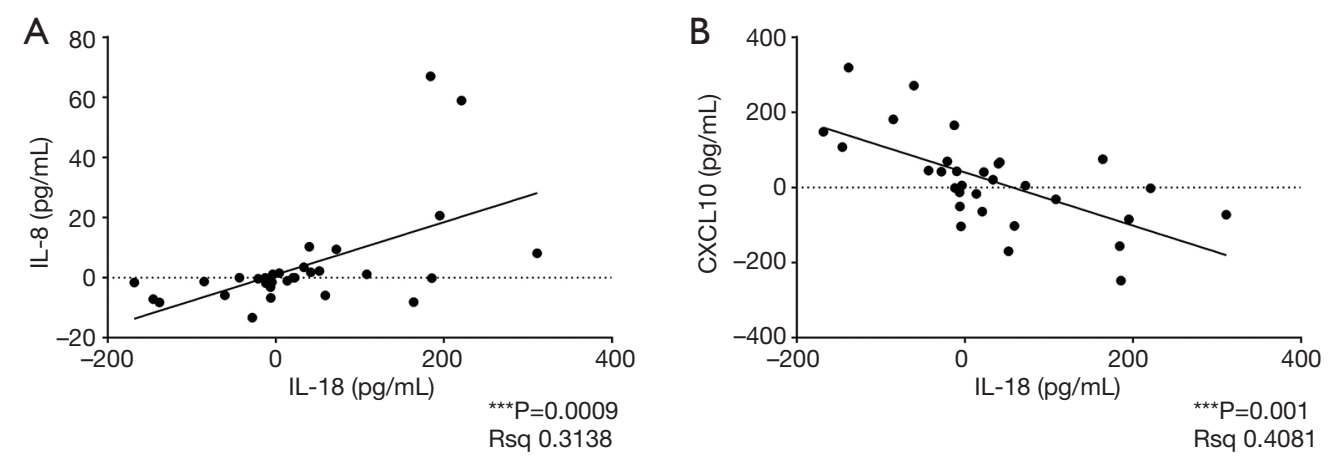

Figure 5 Correlation between different cytokines: IL-18 and IL-8 (A); IL-18 and CXCL10 (B). The horizontal and vertical coordinates represent changes in cytokine levels from pretreatment to posttreatment respectively. Data were examined by the linear regression analysis.

A

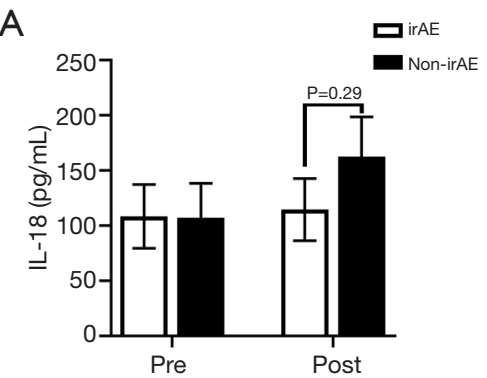

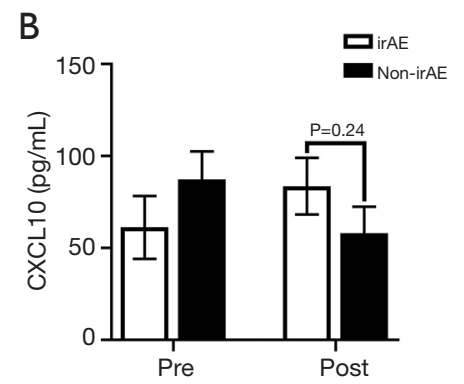

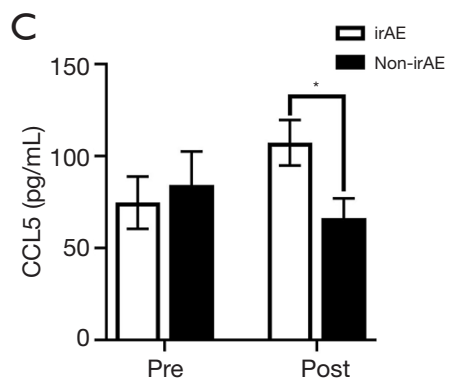

Figure 6 Association between plasma cytokines level and irAEs. The plasma IL-18 (A), CXCL10 (B) and CCL5 (C) levels were assessed between irAE and non-irAE patients at baseline and after 2 cycles of anti-PD-1/PD-L1 treatment. *, $\mathrm{P}<0.05$.

NSCLC (13). The findings were similar to those reported in this study, supporting the reliability of our results. It has also been reported that low granzyme B at nivolumab initiation is associated with a poor tumor response and poor PFS, while low IL-2 and high IFN- $\gamma$ levels are associated with grade 3-4 irAEs in NSCLC patients (14). Moreover, IFN- $\gamma$, TNF- $\alpha$ and IL- 8 levels increased in the sera of lung cancer patients in response to anti-PD-1 therapy (13). Similarly, in our study, IFN- $\gamma$, TNF- $\alpha$ and IL-8 levels increased after anti-PD-1/PD-L1 therapy, even though no significant differences were observed, perhaps because of differences in the population, experimental conditions and/or sample size.

Correlation analysis revealed that the higher the baseline plasma IL-18 level was, the more likely the patients were to benefit from anti-PD-1/PD-L1 treatment, revealing that the level of IL-18 was correlated positively with the tumor response. Indeed, IL-18 plays a pivotal role in inflammation and immune responses. Evidence for the IL-18-mediated anticancer effects involves natural killer (NK) or T cells, and the increased production of IFN- $\gamma$ is usually observed with when high doses of IL-18 are combined with IL-2 or IL-12 $(15,16)$. A high concentration of IL-18 might contribute to the expansion of $\mathrm{IL}-18 \mathrm{R}^{+} \mathrm{CD} 8^{+} \mathrm{T}$ cells within the tumor microenvironment of lung cancer patients, further promoting the $\mathrm{CD}^{+} \mathrm{T}$ cell response in the tumor milieu. Therefore, IL-18 functions as a boosting cytokine for immune checkpoint antagonists in the restoration of $\mathrm{T}$ cell responses $(17,18)$. Furthermore, we found that IL18 plasma levels decreased in patients who achieved PR following anti-PD-1/PD-L1 treatment. One possible reason for this finding is that IL-18 produced by tumor cells promotes tumor progression in a PD-1-dependent manner, thereby blocking PD-1/PD-L1 axis-inhibited tumor cells and resulting in a decrease in IL-18 levels (19). Regardless, we did not find a significant association between PFS and IL-18 levels, possibly because of the limited sample size. Based on the present data, we suggest that IL-18 may be a predictive marker indicating partial remission but not survival for these patients. 
CXCL10 is a small secretory protein in the cysteine-X amino acid-cysteine (CXC) subfamily of cytokines that mediates leukocyte trafficking, adaptive immunity, inflammation, hematopoiesis and angiogenesis by binding to and signaling through the CXCR3 receptor (20). Accumulating evidence suggests a beneficial role for CXCL10 produced by tumor or immune cells, which can recruit $\mathrm{CXCR}^{+}$tumor-infiltrating $\mathrm{T}$ cells and $\mathrm{NK}$ cells associated with tumor suppression (21). Nonetheless, CXCL10 has been shown to enhance migration, invasion, proliferation, survival, and ECM adhesion in diverse cancer cells (22). Importantly, CXCL10 is induced in pancreatic cancer cells, and recruits immunosuppressive CXCR3 ${ }^{+}$ Tregs to pancreatic ductal adenocarcinoma to block the stimulation of $\mathrm{T}$ effector cells by antigen presenting cells or to directly inhibit effector $\mathrm{T}$ and NK cells, favoring pancreatic tumor growth and progression (23). These findings show that CXCL10 facilitates the trafficking of CXCR3-expressing Tregs toward tumor sites, which augments their own production and then promotes cancer outgrowth and poor OS. This may explain why CXCL10 levels showed an inverse correlation with the tumor response and PFS and were increased in patients who benefited from PD-1/PD-L1 inhibitors.

In addition, we found a correlation between two combinations of IL-18 and IL-8, or CXCL10. A previous report revealed that in responding patients, serum IL-8 levels significantly decreased between baseline and the best response but increased upon tumor progression, and early decreases in serum IL-8 levels were associated with prolonged OS in melanoma and NSCLC patients, suggesting that changes in serum IL-8 and IL-18 levels could be used to monitor and predict clinical benefits from immune checkpoint blockade in NSCLC patients (24). A recent study showed that elevated baseline serum IL-8 levels were associated with poor outcomes in squamous and nonsquamous NSCLC patients treated with nivolumab and/or ipilimumab, revealing that serum IL-8 levels might be an independent biomarker to identify unfavorable tumor immunobiology in NSCLC patients receiving immune checkpoint inhibitors (25). Similarly, our study also showed that serum IL-8 levels decreased in patients who benefited from anti-PD-1/PD-L1 treatment but increased in those with PD.

However, there are limitations to the present study. The sample size was relatively small, which could partly explain why an association with the tumor response was not observed when IL-18 and CXCL10 or IL- 8 were combined. Another limitation of our study was that some of our patients had received first-line chemotherapy prior to PD-1/PD-L1 inhibitors. The impact of conventional chemotherapy on cytokine levels on the background of second-line PD-1 inhibition remains vague. As it is very difficult to obtain fresh biopsy tumors in the second line setting, we were not able to analyze the intratumor expression of IL-18 and CXCL10 in this study. Large-scale studies including tumor cytokine analysis are warranted in the future. Therefore, the results of our study will likely serve as a foundation for future large-scale validation studies evaluating temporal changes in plasma cytokine levels and investigating their associations with the tumor response to anti-PD-1/PD-L1 treatments.

Studies on predictive biomarkers for immunotherapy are developing rapidly. Intratumoral PD-L1 expression, tumor mutation burden and neoantigen burden as well as certain oncogene mutations appear to identify patients who are eligible for anti-PD-1/PD-L1 treatments $(26,27)$. As shown in this study, plasma cytokine level analysis, which serves as a noninvasive, cost-effective and reproducible tool for monitoring patients' clinical response, provided a candidate predictive biomarker for lung cancer immunotherapy with potential clinical application. For such potential predictors, consensus and standardization are necessary for their broad application in future studies. Multiplex detection methods and combinations of biomarkers may provide new strategies for predicting the efficacy of PD-1/PD-L1 inhibitors. Among these strategies, blood cytokine detection might become an important part of the integrated predictive model in the future.

\section{Conclusions}

Although clinical applications of immunotherapy have achieved marked success, the efficacy and responsiveness of these agents varies greatly among lung cancer patients. We found that the absolute plasma concentrations of IL18 and CXCL10 were correlated with the degree of tumor response and that the degree of IL-18, CXCL10 and CCL5 fluctuations were correlated significantly with the objective tumor response to anti-PD-1/PD-L1 immunotherapy. Moreover, patients with high CXCL10 expression had significantly shorter PFS than those with low CXCL10 expression. Our findings indicate that cytokine plasma levels may provide prognostic information and constitute predictive markers of the response to anti-PD-1/PD-L1 immunotherapy in patients with lung cancer. Future largescale studies of these cytokine markers are warranted. 


\section{Acknowledgments}

Funding: The work was supported by Open Project funded by National Key R\&D Program of China (No. 2018YFC0910700), the National Natural Sciences Foundation of China (81974247), the Non-profit Central Research Institute Fund of Chinese Academy of Medical Sciences (2018PT31039) and the Beijing Municipal Administration of Hospitals Incubating Program (Code: PX2020045).

\section{Footnote}

Reporting Checklist: The authors have completed the STROBE reporting checklist. Available at http://dx.doi. org/10.21037/atm-20-1513

Data Sharing Statement: Available at http://dx.doi. org/10.21037/atm-20-1513

Conflicts of Interest: All authors have completed the ICMJE uniform disclosure form (available at http://dx.doi. org/10.21037/atm-20-1513). The authors have no conflicts of interest to declare.

Ethical Statement: The authors are accountable for all aspects of the work in ensuring that questions related to the accuracy or integrity of any part of the work are appropriately investigated and resolved. This study was conducted in accordance with the Declaration of Helsinki (as revised in 2013). The study was approved by the Institutional Ethics Board of Peking Cancer Hospital (No. 2017KT57) and informed consent was obtained from all the patients.

Open Access Statement: This is an Open Access article distributed in accordance with the Creative Commons Attribution-NonCommercial-NoDerivs 4.0 International License (CC BY-NC-ND 4.0), which permits the noncommercial replication and distribution of the article with the strict proviso that no changes or edits are made and the original work is properly cited (including links to both the formal publication through the relevant DOI and the license). See: https://creativecommons.org/licenses/by-nc-nd/4.0/.

\section{References}

1. Dubey S, Powell CA. Update in lung cancer 2008. Am J
Respir Crit Care Med 2009;179:860-8.

2. Siegel R, Ma J, Zou Z, et al. Cancer statistics, 2014. CA Cancer J Clin 2014;64:9-29.

3. Keir ME, Butte MJ, Freeman GJ, et al. PD-1 and its ligands in tolerance and immunity. Annu Rev Immunol 2008;26:677-704.

4. Pardoll DM. The blockade of immune checkpoints in cancer immunotherapy. Nat Rev Cancer 2012;12:252-64.

5. Borghaei H, Paz-Ares L, Horn L, et al. Nivolumab versus Docetaxel in Advanced Nonsquamous Non-Small-Cell Lung Cancer. N Engl J Med 2015;373:1627-39.

6. Garon EB, Rizvi NA, Hui R, et al. Pembrolizumab for the treatment of non-small-cell lung cancer. N Engl J Med 2015;372:2018-28.

7. Rittmeyer A, Barlesi F, Waterkamp D, et al. Atezolizumab versus docetaxel in patients with previously treated non-small-cell lung cancer (OAK): a phase 3, openlabel, multicentre randomised controlled trial. Lancet 2017;389:255-65.

8. Herbst RS, Soria JC, Kowanetz M, et al. Predictive correlates of response to the anti-PD-L1 antibody MPDL3280A in cancer patients. Nature 2014;515:563-7.

9. Zhuo M, Chen H, Zhang T, et al. The potential predictive value of circulating immune cell ratio and tumor marker in atezolizumab treated advanced non-small cell lung cancer patients. Cancer Biomark 2018;22:467-76.

10. Grivennikov SI, Greten FR, Karin M. Immunity, inflammation, and cancer. Cell 2010;140:883-99.

11. Eisenhauer EA, Therasse P, Bogaerts J, et al. New response evaluation criteria in solid tumours: revised RECIST guideline (version 1.1). Eur J Cancer 2009;45:228-47.

12. Teng F, Meng X, Kong L, et al. Progress and challenges of predictive biomarkers of anti PD-1/PDL1 immunotherapy: A systematic review. Cancer Lett 2018;414:166-73.

13. Boutsikou E, Domvri K, Hardavella G, et al. Tumour necrosis factor, interferon-gamma and interleukins as predictive markers of antiprogrammed cell-death protein-1 treatment in advanced non-small cell lung cancer: a pragmatic approach in clinical practice. Ther Adv Med Oncol 2018;10:1758835918768238.

14. Costantini A, Julie C, Dumenil C, et al. Predictive role of plasmatic biomarkers in advanced non-small cell lung cancer treated by nivolumab. Oncoimmunology 2018;7:e1452581.

15. Son YI, Dallal RM, Mailliard RB, et al. Interleukin-18 (IL-18) synergizes with IL-2 to enhance cytotoxicity, interferon-gamma production, and expansion of natural 
killer cells. Cancer Res 2001;61:884-8.

16. Nakanishi K. Unique Action of Interleukin-18 on T Cells and Other Immune Cells. Front Immunol 2018;9:763.

17. Timperi E, Focaccetti C, Gallerano D, et al. IL-18 receptor marks functional CD8(+) T cells in non-small cell lung cancer. Oncoimmunology 2017;6:e1328337.

18. Jia Y, Zang A, Jiao S, et al. The interleukin-18 gene promoter $-607 \mathrm{~A} / \mathrm{C}$ polymorphism contributes to nonsmall-cell lung cancer risk in a Chinese population. Onco Targets Ther 2016;9:1715-9.

19. Terme M, Ullrich E, Aymeric L, et al. IL-18 induces PD1-dependent immunosuppression in cancer. Cancer Res 2011;71:5393-9.

20. Zlotnik A, Yoshie O. The chemokine superfamily revisited. Immunity 2012;36:705-16.

21. Wennerberg E, Kremer V, Childs R, et al. CXCL10induced migration of adoptively transferred human natural killer cells toward solid tumors causes regression of tumor growth in vivo. Cancer Immunol Immunother 2015;64:225-35

22. Lee JH, Kim HN, Kim KO, et al. CXCL10 promotes osteolytic bone metastasis by enhancing cancer outgrowth

Cite this article as: Wang $\mathrm{Y}$, Chen $\mathrm{H}$, Zhang T, Yang $\mathrm{X}$, Zhong J, Wang Y, Chi Y, Wu M, An T, Li J, Zhao X, Dong Z, Wang Z, Zhao J, Zhuo M, Huang J. Plasma cytokines interleukin-18 and $\mathrm{C}-\mathrm{X}-\mathrm{C}$ motif chemokine ligand 10 are indicative of the anti-programmed cell death protein- 1 treatment response in lung cancer patients. Ann Transl Med 2021;9(1):33. doi: 10.21037/atm-20-1513 and osteoclastogenesis. Cancer Res 2012;72:3175-86.

23. Billottet C, Quemener C, Bikfalvi A. CXCR3, a doubleedged sword in tumor progression and angiogenesis. Biochim Biophys Acta 2013;1836:287-95.

24. Sanmamed M, Perez-Gracia J, Schalper K, et al. Changes in serum interleukin-8 (IL-8) levels reflect and predict response to anti-PD-1 treatment in melanoma and non-small-cell lung cancer patients. Ann Oncol 2017;28:1988-95.

25. Schalper KA, Carleton M, Zhou M, et al. Elevated serum interleukin-8 is associated with enhanced intratumor neutrophils and reduced clinical benefit of immunecheckpoint inhibitors. Nature Med 2020;26:688-92.

26. Remon J, Chaput N, Planchard D. Predictive biomarkers for programmed death-1/programmed death ligand immune checkpoint inhibitors in non-small cell lung cancer. Curr Opin Oncol 2016;28:122-9.

27. Evans M, O'Sullivan B, Smith M, et al. Predictive markers for anti-PD-1/PD-L1 therapy in non-small cell lung cancer-where are we? Transl Lung Cancer Res 2018;7:682-90. 


\section{Supplementary}

A

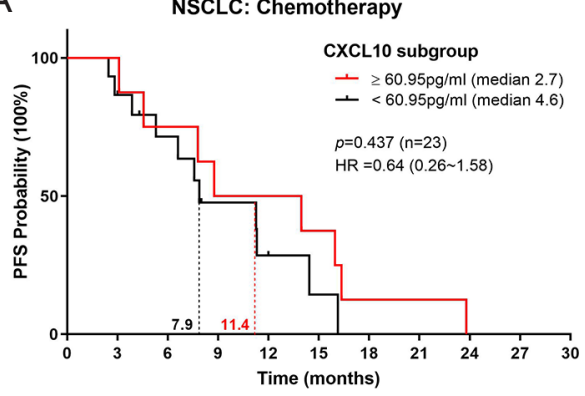

B NSCLC: Chemotherapy

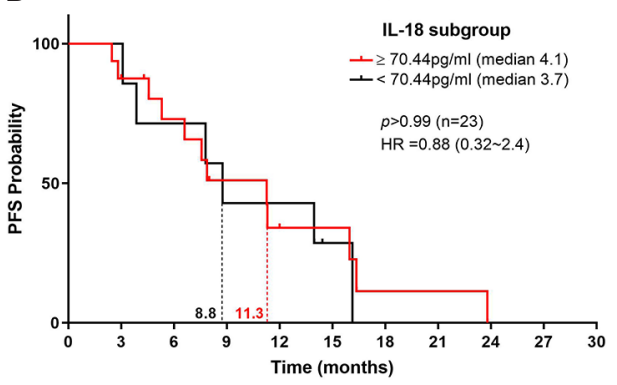

Figure S1 No significant association between IL-18 or CXCL10 level and PFS was observed in the chemotherapy trial arms. The 2-sided log-rank test $\mathrm{P}$ values for the survival analysis, the PFS HR (based on Cox proportional hazard model) between CXCL-10 and IL-18 subgroups are indicated in patients with NSCLC treated with chemotherapy ( $\mathrm{n}=23$ ). (A) Probability of PFS with $\geq 60.95 \mathrm{versus}<60.95 \mathrm{pg} / \mathrm{mL}$ CXCL10 levels. (B) Probability of PFS with $\geq 70.44$ versus $<70.44$ pg/mL IL-18 levels. HR, hazard ratio. 